\title{
Small Distance Behaviour in Field Theory and Power Counting
}

\author{
K. SYMANZIK \\ Deutsches Elektronen-Synchrotron DESY, Hamburg
}

Received May 12, 1970

\begin{abstract}
For infinitesimal changes of vertex functions under infinitesimal variation of all renormalized parameters, linear combinations are found such that the net infinitesimal changes of all vertex functions are negligible relative to those functions themselves at large momenta in all orders of renormalized perturbation theory. The resulting linear first order partial differential equations for the asymptotic forms of the vertex functions are, in quantum electrodynamics, solved in terms of one universal function of one variable and one function of one variable for each vertex function whereby, in contrast to the renormalization group treatment of this problem, the universal function is obtained from nonasymptotic considerations. A relation to the breaking of scale invariance in renormalizable theories is described.
\end{abstract}

\section{Introduction}

The small distance behaviour of Green's and vertex functions in renormalizable quantum field theories has been extensively studied in a formal way via the renormalization group $[1,2]$ and, with equivalent results, some other approaches $[8,9]$. Here we offer an alternative approach to the same problem, which appears to be rather more direct. It leads to formulas that are exact and become the usual asymptotic ones upon a, in principle controllable, neglect.

We study the effect of inserting one extra mass vertex, or a generalized mass vertex in the sense of Wilson [3], into all Feynman diagrams for all vertex functions. (Such vertices are defined as those for which the sum of the mass dimensions of the composing fields, a scalar and the electromagnetic field having dimension one, a spinor field dimension three half, a derivative dimension one, is less than four, e.g. two for a scalar mass vertex and three for a spinor mass vertex.) By such insertion, the superficial divergence $D$ of the corresponding Feynman integral is reduced $^{1}$ (e.g. by two and by one, respectively, for a scalar and a spinor mass vertex). Reduction of the superficial divergence, however, results in decrease of the large-momentum behaviour by the corresponding power of an overall scale factor ${ }^{1}$. This relation between dimension of

\footnotetext{
${ }^{1}$ See, e.g., Ref. [2] p. 321; the index $\omega(G)$ we call $D$. Also ibid., p. 341.
} 
the vertices (i.e., of the terms in the interaction Lagrangian density in the Dyson formula) and large-momentum behaviour is basic in renormalization theory [2] and in many applications (Weinberg sum rules ${ }^{2}$, renormalization of theories with broken symmetry [4-7]).

We now consider, however, the insertion of one such vertex as the infinitesimal operation to the insertion of such interaction term into the Lagrangian density. If this type of vertex is already in the Lagrangian, this change of the theory is expressible as a change of the renormalized masses and coupling constants that completely specify the theory.

Thus, the insertion of one such vertex into all diagrams for a vertex function is equivalent to forming some linear combination of the partial derivatives of that vertex function with respect to all renormalized parameters. The coefficients in this linear combination are independent of momenta or of the vertex function selected, and therefore can be determined by calculating the effect of the insertion operation upon the vertex functions of nonnegative superficial divergence degree, which themselves define, at particular momenta, the renormalized parameters of the theory. Once these coefficients have been determined, we have obtained for every vertex functions at all (in this process, fixed) momenta a linear partial differential equation, the inhomogeneous term being the vertex function with that extra vertex inserted into all diagrams, which is expressible in standard fashion by certain integral operations.

If in such partial differential equation the inhomogeneous term is neglected, we obtain a partial differential equation for the asymptotic form of that vertex function. To see this, we recall from the usual discussion of small distance behaviour $[1,2]$ that asymptotically, all momenta becoming large in the sense $p_{i} \rightarrow \lambda p_{i}, \lambda \rightarrow \infty$, the vertex function behaves, in renormalized perturbation theory, like ${ }^{3} \lambda^{D}$ multiplied by, e.g., in quantum electrodynamics, a double power series in the fine structure constant $\alpha$ and in $\alpha \ln \lambda$, whereby all neglected terms have relative to these one or more factor $\lambda^{-1}$. In the partial differential equation described, by power counting, the inhomogeneous term does have ${ }^{1,3}$ one or more factor $\lambda^{-1}$ relative to all the homogeneous terms, such that the asymptotic form does obey the homogeneous linear partial differential equation.

The solution of this equation is trivial and involves an unknown function, e.g., the value the asymptotic form takes for $\lambda=1$ as a function of the coupling constant. The further discussion of this solution, identical with the one from renormalization group arguments [1,2], is familiar. What has been achieved, for asymptotic formulas, is only that the uni-

${ }^{2}$ See, e.g., the discussion in Ref. [3].

3 The present statements hold in general only if no nontrivial partial sum of momenta vanishes, and conform with Weinberg's results [28], as far as these are applicable, cp. Refs. $[20,29]$. 
versal function $(\psi(z)$ in (II.25) below) of the coupling constant needed in the usual approach $[1,2]$ is here obtained implicitly from the coefficient functions, which were determined without asymptotic considerations. However, in principle, the differential equations are more informative than merely to lead to the usual and, as is well known, essentially useless asymptotic formulas. We elaborate on this point in the discussion. On the other hand, our technique yields, at least directly, only formulas relating to overall scaling, such that other results on asymptotic behaviour, obtained either by more involved uses of the renormalization group [2], by direct asymptotic estimates [8], or by summation of leading logarithmic terms in the perturbation theoretical expansions [9], are so far not covered.

The inhomogeneous partial differential equations allow an amusing application to the problem of the breaking of scale invariance ${ }^{4}$, since they yield, except in theories with scalar or pseudoscalar fields, a new expression for the divergence of the local current associated with scale transformations. The Wilson dimension [3] of this operator is, manifestly, four, such that, as one should anticipate in view of the logarithmic factors in asymptotic formulas, the breaking of scale invariance in renormalizable quantum field theories is not a simple one.

For transparency, we present the method first in Section I applied to the model of a hermitean scalar field in quartic self interaction, and also, because the application, given in the appendix, to the breaking of scale invariance is more interesting in such a model. In Section II, we apply the method to quantum electrodynamics, derive the consequences of current conservation, and obtain familiar [1,2] asymptotic formulas. In the discussion we make remarks on the possible usefulness of our results.

\section{The $\phi^{4}$-Theory}

\section{I.1 Derivation of Partial Differential Equations}

We consider the theory described by the Lagrangian density

$$
L=\frac{1}{2}\left(\partial_{\mu} \phi_{u} \partial^{\mu} \phi_{u}-m_{u}^{2} \phi_{u}^{2}\right)-\frac{1}{24} g_{u} \phi_{u}^{4}+\Delta L\left(\phi_{u}\right)
$$

This expression, containing unrenormalized quantities, is formal and so are the manipulations at the beginning of this section, but the final, renormalized, formulas are correct. A rigorous derivation in the framework of renormalized perturbation theory can be given by first regularizing $[10,11]$ the theory (I.1) and letting the regulator masses go to infinity in the final formulas. For brevity, we omit showing this.

${ }^{4}$ See, e.g., Ref. [3] and references given there. 
The generating functional ${ }^{5}$

$G_{\mathrm{disc}}\{J\}=\sum_{n=0}^{\infty}(n !)^{-1} i^{n} \int \ldots \int d x_{1} \ldots d x_{n} J\left(x_{1}\right) \ldots J\left(x_{n}\right) G_{\mathrm{disc}}\left(x_{1} \ldots x_{n}\right)$

of the renormalized Green's functions

$$
G_{\text {disc }}\left(x_{1} \ldots x_{n}\right)=\left\langle\left(\phi\left(x_{1}\right) \cdots \phi\left(x_{n}\right)\right)_{+}\right\rangle
$$

is for the theory (I.1) (see, e.g., Ref. [27])

$$
G_{\text {disc }}^{\Delta L}\{J\}=\text { const } \exp \left[i \int d x \Delta L\left(-i Z_{\frac{1}{3}}^{\frac{1}{3}} \delta / \delta J(x)\right)\right] G_{\text {disc }}\{J\}
$$

where $G_{\text {disc }}\{J\}$ is the generating functional for the theory (I.1) with $\Delta L$ set zero, and const is such that $G_{\text {disc }}^{A L}\{0\}=1$. We now choose ${ }^{6}$

$$
\Delta L\left(\phi_{u}\right)=-\frac{1}{2} \Delta m_{u}^{2}: \phi_{u}^{2}: .
$$

Then, clearly,

$$
G_{\text {disc }}^{\Delta L}\{J\}\left(m^{2}, g\right)=G_{\text {disc }}\{(1+\Delta z) J\}\left(m^{2}+\Delta m^{2}, g+\Delta g\right)
$$

where $m$ and $g$ are the renormalized mass and coupling constant that specify the theory, and we have taken into account that renormalization also implies an amplitude renormalization convention that will in general not be observed by the Green's functions defined by (I.3).

The infinitesimal form of (I.3), with the choice (I.4), is

$$
\begin{aligned}
\Delta G_{\mathrm{disc}}\{J\}= & \frac{1}{2} i Z_{3} \Delta m_{u}^{2} \\
& \cdot \int d x\left[G_{\mathrm{disc} x x}\{J\}-G_{\mathrm{disc}}\{J\} G_{\mathrm{disc} x x}\{0\}\right]
\end{aligned}
$$

while (I.5) gives

$$
\begin{aligned}
\Delta G_{\mathrm{disc}}\{J\}=\left(\Delta m^{2}\left[\partial / \partial m^{2}\right]\right. & +\Delta g[\partial / \partial g] \\
& \left.+\Delta z \int d x J(x)[\delta / \delta J(x)]\right) G_{\mathrm{disc}}\{J\} .
\end{aligned}
$$

(In (I.6a), subscripts denote ${ }^{5}$ functional derivatives, such that, in view of (I.2),

$$
\left.G_{\mathrm{disc} x_{1} \ldots x_{n}}\{0\}=i^{n} G_{\mathrm{disc}}\left(x_{1} \ldots x_{n}\right) .\right)
$$

5 The concepts and notation used here and later on are described in Ref. [5].

${ }^{6}$ A trilinear term could be dealt with in an analogous manner, but would break the symmetrie $\phi \rightarrow-\phi$ of the undisturbed part in (I.1) such that more renormalization parameters would have to be introduced. Since also the combinatorics with a trilinear term [12] is more complicated and such terms appear also to be of less practical interest, we consider only ordinary mass terms in this paper. 
For connected Green's functions, with generating functional $G\{J\}$ $=\ln G_{\text {disc }}\{J\}$, (I.6) gives

$$
\begin{aligned}
\Delta G\{J\}= & \frac{1}{2} i Z_{3} \Delta m_{u}^{2} \\
& \cdot \int d x\left[G_{x x}\{J\}+G_{x}\{J\} G_{x}\{J\}-G_{x x}\{0\}\right] \\
= & \left(\Delta m^{2}\left[\partial / \partial m^{2}\right]+\Delta g[\partial / \partial g]\right. \\
& \left.+\Delta z \int d x J(x)[\delta / \delta J(x)]\right) G\{J\} .
\end{aligned}
$$

The transition to vertex functions ${ }^{5}$, with generating functional $\Gamma\{\mathscr{A}\}$ such that

$$
\Gamma\left(x_{1} \ldots x_{n}\right)=\Gamma_{x_{1} \ldots x_{n}}\{0\}
$$

is described by

$$
\begin{gathered}
-i G_{x}\{J\}=\mathscr{A}(x), \\
J(x)=i \Gamma_{x}\{\mathscr{A}\}, \\
\Gamma\{\mathscr{A}\}=G\{J\}-i \int d x J(x) \mathscr{A}(x), \\
G_{x y}\{J\}=\Gamma_{x y}^{-1}\{\mathscr{A}\} .
\end{gathered}
$$

In view of (I.8c),

$$
\Delta \Gamma\{\mathscr{A}\}_{\mathscr{A} \text { fixed }}=\Delta G\{J\}_{J \text { fixed }}
$$

such that (I.7) gives

$$
\begin{aligned}
\Delta \Gamma\{\mathscr{A}\}= & \frac{1}{2} i Z_{3} \Delta m_{u}^{2} \\
& \cdot \int d x\left[\Gamma_{x x}^{-1}\{\mathscr{A}\}-\mathscr{A}(x) \mathscr{A}(x)+G(x x)\right] \\
= & \left(\Delta m^{2}\left[\partial / \partial m^{2}\right]+\Delta g[\partial / \partial g]\right. \\
& \left.-\Delta z \int d x \mathscr{A}(x)[\delta / \delta \mathscr{A}(x)]\right) \Gamma\{\mathscr{A}\} .
\end{aligned}
$$

In particular, from (I.9),

and

$$
\begin{aligned}
\Delta \Gamma\left(y_{1} y_{2}\right)= & -i Z_{3} \Delta m_{u}^{2} \delta\left(y_{1}-y_{2}\right) \\
& -\frac{1}{2} i Z_{3} \Delta m_{u}^{2} \int d x d z d u G(x z) G(x u) \Gamma\left(z u y_{1} y_{2}\right) \\
= & \left(\Delta m^{2}\left[\partial / \partial m^{2}\right]+\Delta g[\partial / \partial g]-2 \Delta z\right) \Gamma\left(y_{1} y_{2}\right)
\end{aligned}
$$

$$
\begin{aligned}
& \Delta \Gamma\left(y_{1} y_{2} y_{3} y_{4}\right)=-i Z_{3} \Delta m_{u}^{2} \\
& \cdot \int d x d z d u G(x z) G(x u)\left\{\frac{1}{2} \Gamma\left(z u y_{1} y_{2} y_{3} y_{4}\right)\right. \\
& +\int d v d w G(v w)\left[\Gamma\left(z v y_{1} y_{2}\right) \Gamma\left(w u y_{3} y_{4}\right)\right. \\
& \left.\left.+\Gamma\left(z v y_{1} y_{3}\right) \Gamma\left(w u y_{2} y_{4}\right)+\Gamma\left(z v y_{1} y_{4}\right) \Gamma\left(w u y_{2} y_{3}\right)\right]\right\} \text {. }
\end{aligned}
$$




\section{I.2 Determination of Coefficient Functions}

Determination of $\Delta m^{2}, \Delta g, \Delta z$ in (I.9) requires to fix $\Delta m_{u}^{2}$ by some normalization. We introduce Fourier transforms by

$$
\begin{aligned}
\int d y_{1} \ldots d y_{n} \Gamma\left(y_{1} \ldots y_{n}\right) \exp \left(i p_{1} y_{1}+\cdots+i p_{n} y_{n}\right) & \\
& =(2 \pi)^{4} \delta\left(p_{1}+\cdots+p_{n}\right) \Gamma\left(p_{1} \ldots p_{n}\right)
\end{aligned}
$$

and similarly for the $\Delta \Gamma(\ldots)$, as this notation will not lead to confusion. $\Delta \Gamma(p(-p))$ has, by power counting, superficial divergence degree $D=0$ and, by (I.10), satisfies an inhomogeneous Bethe-Salpeter (BS) equation, with the inhomogeneous term eliminated by one subtraction imposing, as convenient choice,

$$
\left.\Delta \Gamma(p(-p))\right|_{p^{2}=m^{2}}=-i m^{2} .
$$

Thereupon, $\Delta \Gamma(p(-p))$ for all $p$, and all other $\Delta \Gamma(\ldots)$, can be calculated to all orders in renormalized perturbation theory. E.g., the renormalized form of (I.11) is

$$
\begin{aligned}
\Delta \Gamma\left(y_{1} \ldots y_{4}\right)= & \int d z d u d z^{\prime} d u^{\prime} \Delta \Gamma(z u) \\
& \cdot G\left(z z^{\prime}\right) G\left(u u^{\prime}\right)\left\{\frac{1}{2} \Gamma_{2 i}\left(z^{\prime} u^{\prime}, y_{1} y_{2} y_{3} y_{4}\right)\right. \\
& \left.+\int d v d w G(v w)[\operatorname{as~in}(\mathrm{I} .11)]\right\}
\end{aligned}
$$

where $\Gamma_{2 i}\left(z^{\prime} v^{\prime}, y_{1} y_{2} y_{3} y_{4}\right)$ is the vertex function two-particle irreducible ${ }^{7}$ between the first and the second group of arguments and definable by a BS-type integral equation.

For the normalization (I.12), we rewrite (I.9) as

$$
\begin{aligned}
\Delta \Gamma\left(p_{1} \ldots p_{2 n}\right)= & \left(\alpha(g) m^{2}\left[\partial / \partial m^{2}\right]\right. \\
& +\beta(g)[\partial / \partial g]-2 n \gamma(g)) \Gamma\left(p_{1} \ldots p_{2 n}\right) .
\end{aligned}
$$

From the renormalization conditions

$$
\begin{gathered}
\left.\Gamma(p(-p))\right|_{p^{2}=m^{2}}=0, \\
{\left.\left[\partial / \partial p^{2}\right] \Gamma(p(-p))\right|_{p^{2}=m^{2}}=i,} \\
\left.\Gamma\left(p_{1} \ldots p_{4}\right)\right|_{\text {symmetry point }}=-i g,
\end{gathered}
$$

where the symmetry point is defined by $p_{i} p_{j}=\frac{1}{3} m^{2}\left(4 \delta_{i j}-1\right)$, (I.13), and (I.12) we have

$$
\begin{aligned}
-i m^{2}=- & i m^{2} \alpha(g), \\
& {\left.\left[\partial / \partial p^{2}\right] \Delta \Gamma(p(-p))\right|_{p^{2}=m^{2}} } \\
=- & \left.\alpha(g) m^{2}\left[\partial / \partial p^{2}\right]^{2} \Gamma(p(-p))\right|_{p^{2}=m^{2}}-2 i \gamma(g),
\end{aligned}
$$

\footnotetext{
${ }^{7}$ For the concepts and equations used here, see e.g. Refs. [12-14].
} 
and

$$
\begin{aligned}
\left.\Delta \Gamma\left(p_{1} \ldots p_{4}\right)\right|_{\text {symmetry point }} & \\
= & -\left.\frac{1}{2} \alpha(g) \sum_{i=1}^{3} p_{i \mu}\left[\partial / \partial p_{i \mu}\right] \Gamma\left(p_{1} p_{2} p_{3}\left(-p_{1}-p_{2}-p_{3}\right)\right)\right|_{\text {symmetry point }} \\
& \quad-i \beta(g)+4 i g \gamma(g),
\end{aligned}
$$

whereby we have used

$$
m^{2}\left[\partial / \partial m^{2}\right] \Gamma(p(-p))=\left(-p^{2}\left[\partial / \partial p^{2}\right]+1\right) \Gamma(p(-p))
$$

and

$$
\begin{aligned}
\left\{m^{2}\left[\partial / \partial m^{2}\right]\right. & +\frac{1}{2} \sum_{i=1}^{2 n-1} p_{i \mu}\left[\partial / \partial p_{i \mu}\right] \\
& +n-2\} \Gamma\left(p_{1} \ldots p_{2 n-1}\left(-p_{1}-\cdots-p_{2 n-1}\right)\right)=0
\end{aligned}
$$

for $n=2$. We find

$$
\begin{aligned}
& \alpha(g)=1, \\
& \beta(g)=b_{0} g^{2}+b_{1} g^{3}+\cdots, \\
& \gamma(g)=c_{0} g^{2}+c_{1} g^{3}+\cdots,
\end{aligned}
$$

with

$$
\begin{aligned}
& b_{0}=3\left(32 \pi^{2}\right)^{-1} \\
& c_{0}=\left(2^{11} 3 \pi^{4}\right)^{-1} .
\end{aligned}
$$

\section{I.3 Asymptotic Forms of Vertex Functions}

As explained in the introduction, (I.13) yields ${ }^{3}$ for the asymptotic forms of the vertex functions, with (I.16a),

$$
\begin{aligned}
\left(m^{2}\left[\partial / \partial m^{2}\right]\right. & +\beta(g)[\partial / \partial g] \\
& -2 n \gamma(g)) \Gamma_{\text {as }}\left(p_{1} \ldots p_{2 n-1}\left(-p_{1}-\cdots-p_{2 n-1}\right)\right)=0,
\end{aligned}
$$

whose general solution is

$$
\begin{aligned}
\Gamma_{\mathrm{as}}\left(p _ { 1 } \ldots p _ { 2 n - 1 } \left(-p_{1}-\right.\right. & \left.\left.\cdots-p_{2 n-1}\right)\right) \\
& =a(g)^{n} \Phi_{p_{1} \ldots p_{2 n-1}}\left(-\ln m^{2}+\varrho(g)\right)
\end{aligned}
$$

where

$$
a(g)=\exp \left[2 \int_{0}^{g} d g^{\prime} \beta\left(g^{\prime}\right)^{-1} \gamma\left(g^{\prime}\right)\right]
$$


and

$$
\varrho(g)=\int_{g_{0}}^{g} d g^{\prime} \beta\left(g^{\prime}\right)^{-1} .
$$

Thus, for dimensional reasons,

$$
\begin{aligned}
\Gamma_{\text {as }}\left(\lambda p_{1} \ldots \lambda p_{2 n-1}\left(-\lambda p_{1}-\cdots-\lambda p_{2 n-1}\right)\right) \\
=\lambda^{4-2 n} a(g)^{n} \Phi_{p_{1} \ldots p_{2 n-1}}\left(-\ln m^{2}+\ln \lambda^{2}+\varrho(g)\right) .
\end{aligned}
$$

As to scattering amplitudes, (I.20) gives a result only for amplitudes ${ }^{3}$ extrapolated to all $p_{i}^{2}=0$, i.e. for "massless external particles".

The function $\Phi$ in (I.20) expresses the deviation of the asymptotic form of the vertex function from scale invariant form, that is, $\Phi=$ const. Our method, like the, in this respect, equivalent one of the renormalization group [2], gives no information concerning the $\ln \lambda^{2}$-dependence of this function. The only conclusion that can be drawn with some confidence (the flaw being that $\Gamma_{\text {as }}$ may not be asymptotic to $\Gamma$, and may even not exist) from (I.20) is the analog of a famous result of Gell-Mann and Low [1]

$$
\begin{aligned}
\lim _{\lambda \rightarrow \infty} \lambda^{2 n-4} a(g)^{-n} \Gamma_{\text {as }}\left(\lambda p_{1} \ldots \lambda\right. & \left.\lambda p_{2 n-1}\left(-\lambda p_{1}-\cdots-\lambda p_{2 n-1}\right)\right) \\
& =\Phi_{p_{1} \ldots p_{2 n-1}}(\infty) \text { independent of } g .
\end{aligned}
$$

There are relations between the $\Phi$-functions in (I.20), which stem from the renormalized coupled nonlinear integral equations [12-14] among vertex functions. We will not study these relations here. Otherwise, only perturbation theory is available to complement (I.17). One has to calculate $\Gamma_{\text {as }}$ along a noncharacteristic curve in the $m^{2}, g$ plane, the characteristics being the curves $\varrho(g)-\ln m^{2}=$ const. Here, we give the simplest expansion formulas only for the convenience of the reader as their derivation follows familiar patterns. One sets

$$
\Gamma(p(-p))=i p^{2} d\left(\left[p^{2} / m^{2}\right], g\right)^{-1}
$$

and has, from (I.18) with (I.19),

$$
\begin{aligned}
d_{\mathrm{as}}\left(\left[p^{2} / m^{2}\right], g\right)^{ \pm 1}= & \sum_{n=0}^{\infty}(n !)^{-1}\left(\ln \left[p^{2} / m^{2}\right]\right)^{n} \\
& \cdot(\beta(g)[\partial / \partial g] \pm 2 \gamma(g))^{n}\left[d_{\mathrm{as}}(1, g)\right]^{ \pm 1}
\end{aligned}
$$

with the same sign at all three places. Some power series in $g \ln \left(p^{2} / m^{2}\right)$ in these formulas are summed by $\left(\left[p^{2} / m^{2}\right] \equiv x\right)$

$$
\begin{aligned}
a(g) d_{\mathrm{as}}(x, g)= & 1+2 b_{0}^{-1} c_{0} g\left[1-g b_{0} \ln x\right. \\
& \left.+g b_{1} b_{0}^{-1} \ln \left(1-g b_{0} \ln x\right)+g r\right]^{-1}+O\left(g^{3}\right)
\end{aligned}
$$


where we have used (I.16) and

$$
r \equiv-c_{0} b_{0}^{-1}-\frac{1}{2} c_{1} c_{0}^{-1}-\frac{1}{2} b_{0} p_{0} c_{0}^{-1}+\frac{1}{2} b_{1} b_{0}^{-1}
$$

with $p_{0}$ from

$$
d_{\mathrm{as}}(1, g)=1+p_{0} g^{2}+O\left(g^{3}\right),
$$

and in (I.23) it is understood that

$$
1-g b_{0} \ln x>0, \quad\left|g^{-1}-b_{0} \ln x\right|^{-1}=O(g) .
$$

Under similar conditions ${ }^{3}$

$$
\Gamma_{\text {as }}\left(\lambda p_{1} \ldots \lambda p_{3}\left(-\lambda p_{1}-\cdots-\lambda p_{3}\right)\right)=-i g\left(1-2 g b_{0} \ln \lambda\right)^{-1}+O\left(g^{2}\right)
$$

and

$$
\begin{aligned}
& \Gamma_{\text {as }}\left(\lambda p_{1} \ldots \lambda p_{2 n-1}\left(-\lambda p_{1}-\cdots-\lambda p_{2 n-1}\right)\right) \\
& =-i \lambda^{4-2 n} C\left(p_{1} \ldots p_{2 n-1}\right) g^{n}\left(1-2 g b_{0} \ln \lambda\right)^{-n}+O\left(g^{n+1}\right) \quad(n>2)
\end{aligned}
$$

by which formulas the leading logarithms are summed. Final remarks on (I.13) in comparison with the usual approach are made in the discussion.

\section{Quantum Electrodynamics}

\section{II.1 Derivation of Partial Differential Equations}

In analogy to Section I.1, we consider the change of the Lagrangian density by a Fermion mass term. Such a term, if sufficiently carefully defined, observes all invariances of quantum electrodynamics (QED) and, in particular, is invariant ${ }^{8}$ under gauge transformations of the second kind, such that current conservation is not affected. In explicit terms, this means that Ward identities obtain no contribution from such an extra vertex (at zero momentum as needed here, but also at finite momentum) in the Feynman diagrams, a condition that will in turn lead us to the complete definition of that vertex in terms of subtraction prescriptions.

We introduce, for conciseness, the usual anticommuting spinor sources $[15,16] \bar{\eta}(x)$ and $\eta(x)$ along with $J(x)$, whereby we suppress spinor and vector indices wherever expendable. Anticommutators of spinor sources and functional derivatives with respect to them, among themselves and with the spinor fields, all vanish except

$$
\{\delta / \delta \eta(x), \eta(y)\}=\{\delta / \delta \bar{\eta}(x), \bar{\eta}(y)\}=\delta(x-y) .
$$

8 This is related to the fact that the self mass of the electron is gauge invariant. 
We understand all derivatives as left ones, such that e.g.

$$
[\eta(x) \delta / \delta \eta(x), \bar{\psi}(y) \eta(y)]=\bar{\psi}(y) \eta(x) \delta(x-y) .
$$

We use the notation

$$
\begin{aligned}
& \prod_{i=1}^{m}\left[\delta / \delta \bar{\eta}\left(x_{i}\right)\right] \prod_{j=1}^{n}\left[\delta / \delta \eta\left(y_{j}\right)\right] \prod_{k=1}^{l}\left[\delta / \delta J\left(z_{k}\right)\right] G_{\mathrm{disc}}\{\bar{\eta}, \eta, J\} \\
& \equiv G_{\mathrm{disc} x_{1} \ldots x_{m}, y_{1} \ldots y_{n}, z_{1} \ldots z_{l}}\{\bar{\eta}, \eta, J\} \\
&=i^{m-n+l}<\left(\psi\left(x_{1}\right) \ldots \psi\left(x_{m}\right) \bar{\psi}\left(y_{1}\right) \ldots \bar{\psi}\left(y_{n}\right) A\left(z_{1}\right) \ldots A\left(z_{l}\right)\right. \\
&\left.\left.\cdot \exp \left[i \int d x(\bar{\eta}(x) \psi(x)+\bar{\psi}(x) \eta(x)+J(x) A(x))\right]\right)_{+}\right\rangle .
\end{aligned}
$$

(I.3) becomes, with, instead of (I.4),

replaced by

$$
\Delta L\left(\bar{\psi}_{u}, \psi_{u}, A_{u}\right)=-\Delta m_{u} \bar{\psi}_{u} \psi_{u},
$$

$G_{\mathrm{disc}}^{\Delta L}\{\bar{\eta}, \eta, J\}$

$$
=\text { const } \exp \left(-i Z_{2} \Delta m_{u} \int d x\left[\delta^{2} / \delta \eta(x) \delta \bar{\eta}(x)\right]\right) G_{\mathrm{disc}}\{\bar{\eta}, \eta, J\}
$$

and (I.5) by

$$
\begin{aligned}
G_{\text {disc }}^{\Delta L}\{\bar{\eta}, & \eta, J\}(m, e) \\
= & G_{\text {disc }}\left\{\left(1+\Delta z_{2}\right) \bar{\eta},\left(1+\Delta z_{2}\right) \eta,\left(1+\Delta z_{3}\right) J\right\}(m+\Delta m, e+\Delta e) \\
& + \text { gauge correction terms, }
\end{aligned}
$$

whereof the "gauge correction terms" will be discussed later. Infinitesimally, in analogy to (I.6),

and

$$
\begin{aligned}
& \Delta G_{\mathrm{disc}}\{\bar{\eta}, \eta, J\}=-i Z_{2} \Delta m_{u} \\
& \quad \cdot \int d x\left[G_{\mathrm{disc} x, x},\{\bar{\eta}, \eta, J\}-G_{\mathrm{disc}}\{\bar{\eta}, \eta, J\} G_{\mathrm{disc} x, x},\{0,0,0\}\right]
\end{aligned}
$$

$$
\begin{aligned}
\Delta G_{\mathrm{disc}}\{\bar{\eta}, \eta, J\}= & (\Delta m[\partial / \partial m]+\Delta e[\partial / \partial e] \\
& +\int d x\left(\Delta z_{2} \bar{\eta}(x)[\delta / \delta \bar{\eta}(x)]+\Delta z_{2} \eta(x)[\delta / \delta \eta(x)]\right. \\
& \left.\left.+\Delta z_{3} J(x)[\delta / \delta J(x)]\right)\right) G_{\mathrm{disc}}\{\bar{\eta}, \eta, J\} . \\
& + \text { gauge correction terms. }
\end{aligned}
$$

For connected functions, in analogy to (I.7), $\Delta G\{\bar{\eta}, \eta, J\}=-i Z_{2} \Delta m_{u}$

$$
\begin{aligned}
& \int d x\left[G_{x, x},\{\bar{\eta}, \eta, J\}+G_{x,},\{\bar{\eta}, \eta, J\} G_{, x,}\{\bar{\eta}, \eta, J\}-G_{x, x},\{0,0,0\}\right] \\
= & (\text { as in (II.4b)) } G\{\bar{\eta}, \eta, J\}+\text { gauge correction terms. }
\end{aligned}
$$


We now set, in analogy to (I.8),

$$
\begin{gathered}
-i G_{x,,}\{\bar{\eta}, \eta, J\}=\varphi(x), \\
i G_{, x,}\{\bar{\eta}, \eta, J\}=\bar{\varphi}(x), \\
-i G_{,, x}\{\bar{\eta}, \eta, J\}=\mathscr{A}(x), \\
\eta(x)=i \Gamma_{x,,}\{\bar{\varphi}, \varphi, \mathscr{A}\}, \\
\bar{\eta}(x)=-i \Gamma_{, x}\{\bar{\varphi}, \varphi, \mathscr{A}\}, \\
J(x)=i \Gamma_{,, x}\{\bar{\varphi}, \varphi, \mathscr{A}\},
\end{gathered}
$$

$\Gamma\{\bar{\varphi}, \varphi, \mathscr{A}\}=G\{\bar{\eta}, \eta, J\}$

$$
-i \int d x[\bar{\eta}(x) \varphi(x)+\bar{\varphi}(x) \eta(x)+J(x) \mathscr{A}(x)],
$$

whereby in (II.6d-f) a notation analogous to the one described before (II.1) is used, with

$$
\Gamma_{x_{1} \ldots x_{m}, y_{1} \ldots y_{n}, z_{1} \ldots z_{l}}\{0,0,0\}=\Gamma\left(x_{1} \ldots x_{m}, y_{1} \ldots y_{n}, z_{1} \ldots z_{l}\right) .
$$

In analogy to (I.8 d), (see Note added in proof)

$$
\begin{aligned}
G_{x, y}\{\bar{\eta}, \eta, J\} & =-i[\delta / \delta \bar{\eta}(x)] \bar{\varphi}(y) \\
& =-i([\delta / \delta \bar{\varphi}(\cdot)] \bar{\eta}(\cdot))_{x y}^{-1}=\Gamma_{x, y,}^{-1}\{\bar{\varphi}, \varphi, \mathscr{A}\},
\end{aligned}
$$

and $\Gamma(x, y)=,-G^{-1}(x, y$,$) is the negative inverse electron propagator,$ while $\Gamma\left(,, z z^{\prime}\right)=-G^{-1}\left(,, z z^{\prime}\right)$ is the negative inverse photon propagator. The analog of (I.9) is

$$
\begin{aligned}
& \Delta \Gamma\{\bar{\varphi}, \varphi, \mathscr{A}\} \\
& \quad=-Z_{2} \Delta m_{u} d x\left[\Gamma_{x, x}^{-1}\{\bar{\varphi}, \varphi, \mathscr{A}\}-\bar{\varphi}(x) \varphi(x)+G(x, x,)\right]
\end{aligned}
$$

and

$$
\begin{aligned}
\Delta \Gamma\{\bar{\varphi}, \varphi, \mathscr{A}\}= & (\Delta m[\partial / \partial m]+\Delta e[\partial / \partial e] \\
& -\int d x\left(\Delta z_{2} \bar{\varphi}(x)[\delta / \delta \bar{\varphi}(x)]+\Delta z_{2} \varphi(x)[\delta / \delta \varphi(x)]\right. \\
& \left.\left.+\Delta z_{3} \mathscr{A}(x)[\delta / \delta \mathscr{A}(x)]\right)\right) \Gamma\{\bar{\varphi}, \varphi, \mathscr{A}\} \\
& + \text { gauge correction term. }
\end{aligned}
$$

To save space, we do not write down the analoga of (I.10) and (I.11).

\section{II.2 Current Conservation and Determination of Coefficient Functions}

Proceeding as in section I.2, we next discuss the superficial divergence degrees $D$ of the Fourier transforms $\Delta \Gamma\left(p_{1} \ldots p_{m}, q_{1} \ldots q_{n}, k_{1} \ldots k_{l}\right)$. They are all negative except for $m=n=1, l=0$, and for $m=n=0, l=2$, other 
combinations being excluded by gauge invariance of the first kind and/or charge conjugation invariance. Power counting gives $D=0$ for $\Delta \Gamma(p,-p$, and also ${ }^{9}$ for $\Delta \Gamma(,, k(-k))$. Thus it suffices to specify each function at one momentum, to calculate them for all momenta using coupled oncesubtracted BS-equations. Since $\Delta \Gamma(p,-p$, ) is infrared divergent on the mass shell, we set

$$
\Delta \Gamma_{\alpha \beta}(0,0,)=-i m \delta_{\alpha \beta}
$$

which yields the overall normalization for the mass vertex, since

$$
\Delta \Gamma_{\mu \nu}(,, 00)=0
$$

from current conservation similarly as

$$
\Gamma_{\mu v \kappa \lambda}(,, 00000)=0 .
$$

Similarly as in the latter case [17], however, to have (II.9) hold requires to complement the mass vertex by an $A A$-vertex with finite, but integration-convention dependent coefficient. We think that term, which is, besides attention to other invariances, part of the "sufficiently careful definition" mentioned at the beginning of Section II.1, tacitly implied in all previous formulas that indicate the formal definition of the mass vertex. In Gupta-Bleuler gauge and symmetric integration convention [18], the finite term to be subtracted from the unadjusted $\Delta \Gamma_{\mu \nu}(,, k(-k))$ is $i m^{2}\left(4 \pi^{2}\right)^{-1} e^{2} g_{\mu v}$. (See Note added in proof.)

Once (II.9) has been secured, however, $\Delta \Gamma_{\mu v}(,, k(-k))$ is transverse at all momenta, while $\Gamma_{\mu v}(,, k(-k))$ it is added to has also a gaugedependent longitudinal part. This mismatch is the reason for the occurrence of the gauge correction term in (II.1) and, going backwards, all previous equations. We shall find that term by studying the consequences of current conservation generally.

For definiteness, we choose Gupta-Bleuler gauge. We have the familiar Ward-Takahashi identities [19] in the form

$\square \partial_{\mu}\left[\delta / \delta J_{\mu}(z)\right] G_{\mathrm{disc}}\{\bar{\eta}, \eta, J\}$

$$
=\left(-i e \bar{\eta}(z)[\delta / \delta \bar{\eta}(z)]+i e \eta(z)[\delta / \delta \eta(z)]-i \partial_{\mu} J^{\mu}(z)\right) G_{\mathrm{disc}}\{\bar{\eta}, \eta, J\}
$$

wherefrom, for vertex functions, using (II.6),

$$
\begin{aligned}
i \square \partial_{\mu} \mathscr{A}^{\mu}(z)= & (-i e \bar{\varphi}(z)[\delta / \delta \bar{\varphi}(z)] \\
& \left.+i e \varphi(z)[\delta / \delta \varphi(z)]+\partial_{\mu}\left[\delta / \delta \mathscr{A}_{\mu}(z)\right]\right) \Gamma\{\bar{\varphi}, \varphi, \mathscr{A}\} .
\end{aligned}
$$

9 Because of Furry's theorem, there will be precisely one closed loop with an odd number of corners, namely the one containing the mass vertex. Since this vertex has unit Dirac matrix, the highest loop momentum power drops out in the Dirac trace. 
In particular,

$$
\Gamma_{\mu \nu}\left(,, z z^{\prime}\right)_{\text {long }}=i \partial_{\mu} \partial_{\nu} \delta\left(z-z^{\prime}\right)
$$

from the inhomogeneous term in (II.11).

Upon the convention-dependent correction that ensures (II.9), all $\Delta \Gamma($. .) satisfy homogeneous Ward-Takahashi identities, i.e.

$$
\begin{aligned}
(-i e \bar{\varphi}(z)[\delta / \delta \bar{\varphi}(z)] & +i e \varphi(z)[\delta / \delta \varphi(z)] \\
& \left.+\partial_{\mu}\left[\delta / \delta \mathscr{A}_{\mu}(z)\right]\right) \Delta \Gamma\{\bar{\varphi} ; \varphi, \mathscr{A}\}=0,
\end{aligned}
$$

as obtained by applying formally the defining operation (II.7a) on (II.11). A simple calculation shows that (II.7b) and (II.13) are consistent if and only if

and (see Note added in proof)

$$
\Delta e=e \Delta z_{3}
$$

$$
\text { gauge correction term in (II.7b) }=-i \int d z\left[\partial_{\mu} \mathscr{A}^{\mu}(z)\right]^{2} \cdot \Delta z_{3} \text {. }
$$

The gauge correction terms in (II.3) that follow from (II.15) are more complicated and need not be given here. It is not possible to modify the defining linear operation for $\Delta G_{\text {disc }}$, (II.4a), by terms of Wilson [3] dimension $\leqq 3$ in a way that would correspond to a simple renormalized linear operation on $G_{\text {disc }}$ as in (II.4b), since the candidate for the unrenormalized operation is of dimension four. However, all gauge correction terms vanish if one uses the transverse (Landau) gauge where both (II.12) and (II.15) are consistently replaceable by zero.

The intermediate renormalization [20] we adopt ${ }^{10}$ is described by

$$
\begin{gathered}
\left.\Gamma(p,-p,)\right|_{p=m}=0, \\
{\left.\left[\partial / \partial p_{\mu}\right] \Gamma(p,-p,)\right|_{p=0}=i \gamma^{\mu},} \\
\Gamma_{\mu v}(,, 00)=0, \\
{\left.\left[\partial / \partial k_{\alpha}\right] \Gamma_{\mu \nu}(,, k(-k))\right|_{k=0}=0,} \\
{\left.\left[\partial^{2} / \partial k_{\alpha} \partial k_{\beta}\right] \Gamma_{\mu \nu}(,, k(-k))\right|_{k=0}=-2 i g_{\mu \nu} g^{\alpha \beta}}
\end{gathered}
$$

corresponding to

$$
\Gamma_{\mu v}(,, k(-k))=-i k^{2} g_{\mu \nu}+i\left(k^{2} g_{\mu \nu}-k_{\mu} k_{v}\right) O\left(k^{2}\right) .
$$

${ }^{10}$ This formulation of the renormalization used in Ref. [20] has been proposed by $\mathrm{K}$. Hepp (private communication). 
The identities (II.11) supply (II.10) and

$$
\Gamma_{\mu}(0,0,0)=-i e \gamma_{\mu} .
$$

For the normalization (II.8), we rewrite (II.7b) explicitly in Fourier space as (see Note added in proof)

$$
\begin{aligned}
& \Delta \Gamma\left(p_{1} \ldots p_{n}, q_{1} \ldots q_{n}, k_{1} \ldots k_{l}\right)=(\alpha(e) m[\partial / \partial m] \\
& \left.\quad+\gamma_{3}(e)[e(\partial / \partial e)-l]-2 n \gamma_{2}(e)\right) \Gamma\left(p_{1} \ldots p_{n}, q_{1} \ldots q_{n}, k_{1} \ldots k_{l}\right) \\
& \quad+\gamma_{3}(e) i\left(k_{1 \mu} k_{2 v}+k_{1 v} k_{2 \mu}\right) \delta_{n 0} \delta_{l 2} .
\end{aligned}
$$

Using also

$$
\begin{aligned}
m[\partial / \partial m] & \Gamma\left(p_{1} \ldots p_{n}, q_{1} \ldots q_{n}, k_{1} \ldots k_{l-1}\left(-p_{1}-\cdots-k_{l-1}\right)\right) \\
= & \left(-\sum_{i=1}^{n} p_{i \mu}\left[\partial / \partial p_{i \mu}\right]-\sum_{i=1}^{n} q_{i \mu}\left[\partial / \partial q_{i \mu}\right]-\sum_{i=1}^{l-1} k_{i \mu}\left[\partial / \partial k_{i \mu}\right]+4-3 n-l\right) \\
& \cdot \Gamma\left(p_{1} \ldots p_{n}, q_{1} \ldots q_{n}, k_{1} \ldots k_{l-1}\left(-p_{1}-\cdots-k_{l-1}\right)\right)
\end{aligned}
$$

we have from (II.16-18) (see Note added in proof)

$$
\begin{gathered}
\Delta \Gamma(0,0,)=\left(\alpha(e)+\gamma_{3}(e) e[\partial / \partial e]-2 \gamma_{2}(e)\right) \Gamma(0,0,), \\
{\left.\left[\partial / \partial p_{\mu}\right] \Delta \Gamma(p,-p,)\right|_{p=0}=-2 i \gamma_{2}(e) \gamma^{\mu},} \\
\Delta \Gamma_{\mu v}(,, 00)=0, \\
{\left.\left[\partial / \partial k_{\alpha}\right] \Delta \Gamma_{\mu \nu}(,, k(-k))\right|_{k=0}=0,} \\
{\left.\left[\partial^{2} / \partial k_{\alpha} \partial k_{\beta}\right] \Delta \Gamma_{\mu \nu}(,, k(-k))\right|_{k=0}} \\
=2 i \gamma_{3}(e)\left(2 g_{\mu v} g^{\alpha \beta}-g_{\mu}^{\alpha} g_{v}^{\beta}-g_{\mu}^{\beta} g_{v}^{\alpha}\right), \\
\Delta \Gamma_{\mu}(0,0,0)=2 i \gamma_{2}(e) e \gamma_{\mu}, \\
\Delta \Gamma_{\mu v \kappa \lambda}(,, 0000)=0 .
\end{gathered}
$$

While (II.20c) is (II.9) and has been discussed before, (II.20d) is trivial, and (II. $20 \mathrm{~g}$ ) is satisfied and yields no further information. The identities (II.13) secure consistency of (II.20b) with (II.20f). From the remaining equations we find

$$
\begin{gathered}
\alpha(e)=1+O\left(e^{2}\right), \\
\gamma_{2}(e)=\left(8 \pi^{2}\right)^{-1} e^{2}+O\left(e^{4}\right), \\
\gamma_{3}(e)=\left(12 \pi^{2}\right)^{-1} e^{2}+O\left(e^{4}\right) .
\end{gathered}
$$

While these functions are gauge dependent, it follows e.g. from its relation to the asymptotic forms of the (gauge independent) pure photon vertex functions that the ratio $\gamma_{3}(e) / \alpha(e)$ is gauge independent, and also the identity (II.14) holds in all gauges. 


\section{II.3 Asymptotic Form of Vertex Functions}

For the two-photon vertex function, we set

$$
\Gamma_{\mu v}(,, k(-k))=i\left(-g_{\mu v} k^{2}+k_{\mu} k_{v}\right) d\left(\left[k^{2} / m^{2}\right], e^{2}\right)^{-1}-i k_{\mu} k_{v} .
$$

As in Section I.3, the step justified in the introduction gives from (II.18)

$$
\left(m^{2}\left[\partial / \partial m^{2}\right]+\alpha(e)^{-1} e^{2} \gamma_{3}(e)\left[e^{-2}+\left(\partial / \partial e^{2}\right)\right]\right) d_{\mathrm{as}}\left(\left[k^{2} / m^{2}\right], e^{2}\right)=0
$$

or, with $e^{2} \gamma_{3}(e) / \alpha(e)=\sigma\left(e^{2}\right)$,

$$
\left(-k^{2}\left[\partial / \partial k^{2}\right]+\sigma\left(e^{2}\right)\left[\partial / \partial e^{2}\right]\right)\left[e^{2} d_{\mathrm{as}}\left(\left[k^{2} / m^{2}\right], e^{2}\right)\right]=0 .
$$

With $e^{2}=\alpha$ and $k^{2} / m^{2}=x$, the general solution of (II.22) is

where

$$
\alpha d_{\text {as }}(x, \alpha)=\Phi(\ln x+\varrho(\alpha))
$$

$$
\varrho(\alpha)=\int_{\alpha_{0}}^{\alpha} d \alpha^{\prime} \sigma\left(\alpha^{\prime}\right)^{-1}
$$

Writing (II.23) as

$$
\ln x=-\varrho(\alpha)+\Phi^{-1}\left(\alpha d_{\mathrm{as}}(x, \alpha)\right)
$$

we compare it with the formula ${ }^{11}$ of Gell-Mann and Low

$$
\ln x=\int_{\alpha d_{\mathrm{as}}(1, \alpha)}^{\alpha d_{\mathrm{as}}(x, \alpha)} d z \psi(z)^{-1} \equiv \Psi\left(\alpha d_{\mathrm{as}}(x, \alpha)\right)-\Psi\left(\alpha d_{\mathrm{as}}(1, \alpha)\right) .
$$

Thus, the present method supplies implicitly the function $\psi$ or $\Psi$ if $d_{\mathrm{as}}(1, \alpha)$ is known since $\varrho(\alpha)$ was determined by relatively rigorous nonasymptotic considerations.

In practical terms, the difference is that in the series

$$
d_{\mathrm{as}}(x, \alpha)=1+\alpha\left(a_{10}+a_{11} \ln x\right)+\alpha^{2}\left(a_{20}+a_{21} \ln x+a_{22}[\ln x]^{2}\right)+\cdots
$$

(or in the analogous series for $d_{\mathrm{as}}(x, \alpha)^{-1}$ ) in the present method only the $a_{n 0}$ need be calculated, while in the usual procedure $[2,21,22]$ one needs more coefficients to construct, implicitly, also the power series for $\psi(z)$. Since, apparently, calculation of the $a_{n 0}$ requires calculation also of all $a_{n k}, k>0$, there is no practical gain. However, in principle our

${ }^{11}$ Formula (5.9) of Ref. [1] 
method appears to be superior, as we will argue in the discussion. We refrain from deriving from (II.18) further asymptotic formulas as these are identical with known [2] renormalization group results.

\section{Discussion}

The present method to derive asymptotic formulas for vertex functions from power counting arguments alone appears to be more direct than the usual one [2] based on the renormalization group, where the correctness of certain statements on qualitative subtraction parameter dependence is not easily [21] established. Moreover, due to the welldefined left hand sides in (I.13) and (II.18), in principle, corrections to the asymptotic forms could be calculated.

More important is the fact that (I.13) and (II.18) involve the vertex functions themselves rather than their relatively artificial asymptotic forms, such that information on e.g. initial data, for the asymptotic forms most difficult to obtain, to supplement the partial differential equations, could be sought from nonperturbation theoretical sources. No analysis of (I.13) or (II.18) along such lines has been attempted in this paper.

In fact, the asymptotic forms (I.18), and similarly for QED, of the vertex functions are explicitly expressible by a simple modification, which is justified on the basis of the large-momentum behaviour of $\Delta \Gamma$ discussed in the Introduction, of the elementary solution formulas for the inhomogeneous equations (I.13) and (II.18).

Our formula (I.18) and its QED analog relate only to overall scaling, while already known techniques $[2,8,9]$ allow more detailed statements. However, the relations, mentioned after (I.21), between the $\Phi$-functions have not been exploited here and do supply more information in those cases where the dominant contributions to asymptotic behaviour come from the integration region of large momenta ${ }^{12}$ in the nonlinear integral equations of Refs. [12-14]. (See Note added in proof.)

Finally, we remark that formulas (I.13) and (II.18), and their analoga in other renormalizable theories, could be used to complement the renormalization rules of Ward [23], whereupon this scheme would give results identical with those obtained on the basis of Bogoliubov-ParasiukHepp $[2,24]$ renormalization theory.

Formulas analogous to (A.5), (A.7) and (A.8) in more realistic theories could be useful in phenomenological discussions of the breaking of scale invariance.

Acknowledgement. The presentation in the appendix was strongly influenced by the author's earlier discussions with and an important private communication from S. Coleman. The author is much indebted to S. Coleman for these discussions and the communication.

${ }^{12}$ Cp. the technique of Ref. [8] and the discussion of integration regions in Ref. [9]. 


\section{Appendix}

The Breaking of Scale Invariance

The current $S^{\mu}(x)$ associated with scale transformations ${ }^{13}$ is defined such that its Green's functions ${ }^{5}$ satisfy the Ward identities

$$
\begin{aligned}
\partial_{\mu}\left\langle\left( S^{\mu}(x) A\left(y_{1}\right)\right.\right. & \left.\left.\ldots A\left(y_{n}\right)\right)_{+}\right\rangle \\
= & \left\langle\left(\left[\partial_{\mu} S^{\mu}(x)\right] A\left(y_{1}\right) \ldots A\left(y_{n}\right)\right)_{+}\right\rangle \\
& -i \sum_{i=1}^{n} \delta\left(x-y_{i}\right)\left(d_{i}+y_{i}^{v} \partial_{i v}\right)\left\langle\left(A\left(y_{1}\right) \ldots A\left(y_{n}\right)\right)_{+}\right\rangle
\end{aligned}
$$

where $d_{i}$ is the mass dimension of the $i^{\text {th }}$ field operator, with argument $y_{i}(d=1$ for scalar and vector fields, $d=3 / 2$ for spinor fields). Integration of (A.1) gives, if there are no massless particles in the theory that could give rise to nonvanishing boundary terms,

For dimensional reasons,

$$
\begin{aligned}
& \int d x\left\langle\left(\left[\partial_{\mu} S^{\mu}(x)\right] A\left(y_{1}\right) \ldots A\left(y_{n}\right)\right)_{+}\right\rangle \\
&=i \sum_{i=1}^{n}\left(d_{i}+y_{i}^{v} \partial_{i v}\right)\left\langle\left(A\left(y_{1}\right) \ldots A\left(y_{n}\right)\right)_{+}\right\rangle .
\end{aligned}
$$

$$
\left(\sum_{i=1}^{n} y_{i}^{v} \partial_{i v}-m[\partial / \partial m]+\sum_{i=1}^{n} d_{i}\right) \cdot\left\langle\left(A\left(y_{1}\right) \ldots A\left(y_{n}\right)\right)_{+}\right\rangle=0
$$

provided all masses are kept in fixed proportion to $m$. Thus,

$$
\begin{aligned}
\int d x\left\langle\left(\left[\partial_{\mu} S^{\mu}(x)\right] A\left(y_{1}\right) \ldots A\left(y_{n}\right)\right)_{+}\right\rangle & \\
& =2 \operatorname{im}^{2}\left[\partial / \partial m^{2}\right]\left\langle\left(A\left(y_{1}\right) \ldots A\left(y_{n}\right)\right)_{+}\right\rangle .
\end{aligned}
$$

If we regularize $[10,11]$ the theory such that the Lagrangian expression is meaningful, and keep also the regulator masses in fixed proportion to $m$ such that (A.2) remains valid, we can evaluate the right hand side of (A.2) using the Schwinger action principle [15,27], with the result

$$
\begin{aligned}
& \int d x\left\langle\left(\left[\partial_{\mu} S^{\mu}(x)\right] A\left(y_{1}\right) \ldots A\left(y_{n}\right)\right)_{+}\right\rangle \\
& =-2 m^{2} \int d x\left\langle T\left(\left[\left(\partial L(x) / \partial m^{2}\right)-\left\langle\left(\partial L(x) / \partial m^{2}\right)\right\rangle\right] A\left(y_{1}\right) \ldots A\left(y_{n}\right)\right)\right\rangle .
\end{aligned}
$$

For definiteness, we now consider the theory (I.1) (with $\Delta L=0$ ), but will, for brevity, omit to indicate the regularization explicitly. Then, in (A.3),

$$
m^{2}\left(\partial L(x) / \partial m^{2}\right)=m_{u}^{2}\left(\partial L(x) / \partial m_{u}^{2}\right)=-\frac{1}{2} m_{u}^{2} \phi_{u}(x)^{2} .
$$

\footnotetext{
${ }^{13}$ See, e.g., Ref. $[3,25,26]$ and earlier references given there.
} 
However, that $\phi_{u}(x)^{2}$ is only logarithmically but $m_{u}^{2}$ quadratically divergent indicates that the regularized form of (A.4) will involve complicated cancellations of (in the regularization limit) quadratically divergent expressions. While it is clear from (A.2) and (A.3) that, at least in the space-time integration, all divergences will be removed, (A.4) yields an inconvenient form for the rigth hand side of (A.3).

Therefore, it is suggestive to use (I.6b) in (A.2). With the normalization (I.12) of $\Delta m_{u}^{2}$, we obtain for the right hand side of (A.2), in the theory (I.1), using again the action principle,

$$
\begin{aligned}
\int d x & \left\langleT \left(\left\{ Z_{3} \Delta m_{u}^{2} \phi(x)^{2}+2 \beta(g)[\partial L(x) / \partial g]\right.\right.\right. \\
& \left.\left.\left.-2 i n \gamma(g)+i n \beta(g)\left[\partial \ln Z_{3} / \partial g\right]-\text { vac. exp. val. }\right\} \phi\left(y_{1}\right) \ldots \phi\left(y_{n}\right)\right)\right\rangle .
\end{aligned}
$$

Here we use

$$
\begin{aligned}
i n\left\langle T\left(\phi\left(y_{1}\right) \ldots \phi\left(y_{n}\right)\right)\right\rangle= & i \int d x \sum_{i=1}^{n} \delta\left(x-y_{i}\right)\left\langle T\left(\phi\left(y_{1}\right) \ldots \phi\left(y_{n}\right)\right)\right\rangle \\
= & \frac{1}{2} \int d x\left\{\left\langle T\left(\left[\square \phi_{u}(x)^{2}\right] \phi\left(y_{1}\right) \ldots \phi\left(y_{n}\right)\right)\right\rangle\right. \\
& \left.-\square_{x}\left\langle T\left(\phi_{u}(x)^{2} \phi\left(y_{1}\right) \ldots \phi\left(y_{n}\right)\right)\right\rangle\right\},
\end{aligned}
$$

where in the curly bracket the second term may be omitted,

$$
\frac{1}{2} \square \phi_{u}(x)^{2}=\partial_{\mu} \phi_{u}(x) \partial^{\mu} \phi_{u}(x)+\phi_{u}(x)\left[-m_{u}^{2} \phi_{u}(x)-\frac{1}{6} g_{u} \phi_{u}(x)^{3}\right],
$$

and

$$
\partial L(x) / \partial g=-\frac{1}{2} \phi_{u}(x)^{2}\left(\partial m_{u}^{2} / \partial g\right)-\frac{1}{24} \phi_{u}(x)^{4}\left(\partial g_{u} / \partial g\right) .
$$

Altogether, (A.5) becomes

$$
\begin{aligned}
& \int d x\left\langle\left(\left[\partial_{\mu} S^{\mu}(x)\right] \phi\left(y_{1}\right) \ldots \phi\left(y_{n}\right)\right)_{+}\right\rangle \\
& =\int d x\left\langleT \left(\left\{ Z_{3} \Delta m_{u}^{2} \phi(x)^{2}-\frac{1}{2} \beta(g)\left[\partial\left(Z_{3} m_{u}^{2}\right) / \partial g\right] \phi(x)^{2}\right.\right.\right. \\
& \quad-\frac{1}{24} \beta(g)\left[\partial\left(Z_{3}^{2} g_{u}\right) / \partial g\right] \phi(x)^{4}+\left[\beta(g)\left(\partial Z_{3} / \partial g\right)-2 Z_{3} \gamma(g)\right] \\
& . \partial_{\mu} \phi(x) \partial^{\mu} \phi(x)+2 \gamma(g)\left[Z_{3} m_{u}^{2} \phi(x)^{2}+\frac{1}{6} Z_{3}^{2} g_{u} \phi(x)^{4}\right] \\
& \left.\left.\quad \text {-vac. exp. val. }\} \phi\left(y_{1}\right) \ldots \phi\left(y_{n}\right)\right)\right\rangle .
\end{aligned}
$$

We examine the terms in the curly bracket. The first term is finite by construction since by finiteness we understand the one of the Green's functions. The second term is to order $g^{2}$ (cp. (I.16)) simply proportional to a quadratic divergence that is, however, to this order precisely (i.e., also in its regularized form) cancelled by the divergent term separated from the third term by converting it into a Wick product. The fourth and fifth term are finite to order $g^{2}$. Thus, while in (A.3) with (A.4) the problem of correct cancellation of divergences arises already in order $g$, with (A.7) it appears not before order $g^{3}$. 
The right hand side of (A.7) makes it evident that scale invariance is broken by terms of Wilson [3] dimension four ${ }^{14}$, since, with (I.16),

$$
\begin{aligned}
& \{\} \text { of (A.7) }=\text { finite term proportional: } \phi(x)^{2}: \\
& \qquad-\frac{1}{24} b_{0} g^{2}: \phi(x)^{4}:-2 c_{0} g^{2}: \partial_{\mu} \phi(x) \partial^{\mu} \phi(x):+O\left(g^{3}\right) .
\end{aligned}
$$

However, (A.8) cannot be identified with $\partial_{\mu} S^{\mu}(x)$. The reason is that the steps that led to (A.7) are meaningful only in the regularized theory, and for that theory, if on the right hand side of (A.6) the square of the regularized field is used, that side is zero, such that the identification contemplated is possible only up to a term proportional to : $\square \phi(x)^{2}$ : that cannot be specified by an analysis based only on the integrated form (A.2) of (A.1). Moreover, $S^{\mu}(x)$ and the energy-momentum tensor $\Theta^{\mu v}(x)$ it is related to [26] by $S^{\mu}(x)=x_{v} \Theta^{\mu \nu}(x)$ do have a corresponding ambiquity, formally expressible by

$$
\Theta^{\mu v}(x) \rightarrow \Theta^{\mu v}(x)+\lambda\left(g^{\mu v} \square-\partial^{\mu} \partial^{v}\right) \phi(x)^{2},
$$

because (A.1) specifies $S^{\mu}(x)$ incompletely without independent characterization of $\partial_{\mu} S^{\mu}(x)$.

This ambiguity can be exploited to make $\Theta^{\mu \nu}(x)$ and $S^{\mu}(x)$ renormalizable operators ${ }^{15}$. However, the terms of dimension four in (A.8) cannot, of course, thereby be removed.

In QED, where no such ambiguity arises [26], the electron self mass is only logarithmically divergent, but a (non-gauge-invariant counter) term in the Lagrangian density of the form "square of the vector potential multiplied by a quadratically divergent coefficient" similar to (A.4) leads also here, as in all renormalizable theories, to a breaking of scale invariance by terms of dimension four, as one should anticipate in view of the logarithmic terms that multiply scale invariant expressions for vertex functions (cp. Sections I.3 and II.3) at large momenta.

Note added in proof. Formula (II.6h) is oversimplified, the matrix inversion involved being extensive. - The adjustment term discussed after (II.10) is nonzero also in higher orders, and in these logarithmically divergent. (The author thanks R. A. Brandt for a discussion.) - Insertion of a mass vertex into all vertex functions in QED results in such infinitesimal change of gauge that the change of the longitudinal part of the photon propagator due to change of the amplitude renormalization factor is precisely compensated. (II.15) and consequently (II.18) and (II.20) are incomplete, and the coefficient functions (II.21) need be calculated for varying gauge. The transverse (Landau) gauge, the only one stable under mass vertex insertion, has in (II.21b) $\gamma_{2}(e)=O\left(e^{4}\right)$. The correct gauge terms in (II.15) and (II.18) for arbitrary gauges will be given elsewhere. - The integral relations between $\Phi$ functions mentioned in the Discussion are the usual ones for vertex functions [12-14] but for the (non-scale invariant) theory with massless particles.

${ }^{14}$ The observation that the dimension of the divergence of the scaling current in the scalar model is four was privately communicated to the author by S. Coleman.

${ }^{15} \mathrm{Cp}$., in this connection, Ref. [26]. 


\section{References}

1. Gell-Mann, M., Low, F. E.: Phys. Rev. 95, 1300 (1954).

2. Bogoliubov, N. N., Shirkov, D. V.: Introduction to the Theory of Quantized Fields. New York: Interscience Publ. 1959.

3. Wilson, K.: Phys. Rev. 179, 1499 (1969).

4. Gell-Mann, M., Zachariasen, F.: Phys. Rev. 123, 1065 (1961).

5. Symanzik, K.: Commun. Math. Phys. 16, 48 (1970).

6. - Coral Gables Conference on Fundamental Interactions at High Energy II, p. 263, Eds. A. Perlmutter, G. J. Iverson, R. M. Williams, New York: Gordon and Breach, 1970.

7. Stora, R., Symanzik, K.: (in preparation).

8. Landau, L. D., Abrikosov, A., Halatnikov, L.: Suppl. al Nuovo Cimento 3, 80 (1956).

9. Appelquist, T., Primack, J. R.: Phys. Rev. 1D, 1144 (1970).

10. Pais, A., Uhlenbeck, G. E.: Phys. Rev. 79, 145 (1950).

11. Gupta, S. N.: Proc. Phys. Soc. (London) A 66, 129 (1953).

12. Johnson, R. W.: J. Math. Phys. 10, (to appear).

13. Symanzik, K.: In: Lectures on High Energy Physics, Ed. B. Jakšić, Zagreb: 1961. Reprinted. New York: Gordon and Breach 1966.

14. Taylor, J. G.: Suppl. al Nuovo Cimento 1, 857 (1963).

15. Schwinger, J.: Proc. Natl. Acad. Sci. U.S. 37, 452, 455 (1951).

16. - Proc. Natl. Acad. Sci. U.S. 48, 603 (1962).

17. Brandt, R. A.: Ann. Phys. (N. Y.) 52, 122 (1969).

18. Jauch, J. M., Rohrlich, F.: The Theory of Photons and Electrons. Cambridge (Mass.): Addison-Wesley 1955.

19. Kazes, E.: Nuovo Cimento 13, 1226 (1959).

20. Bjorken, J. D., Drell, S. D.: Relativistic Quantum Fields. New York: McGraw-Hill 1965.

21. Eriksson, K. E.: Nuovo Cimento 27, 178 (1963).

22. Jackiw, R.: Nucl. Phys. B 5, 158 (1968).

23. Ward, J. C.: Phys. Rev. 84, 897 (1951).

24. Hepp, K.: Commun. Math. Phys. 2, 301 (1966).

25. Gross, D. J., Wess, J.: Phys. Rev., (to appear).

26. Callan Jr., C. G., Coleman, S., Jackiw, R.: Ann. Phys. (N. Y.), (to appear).

27. Lam, C. S.: Nuovo Cimento 38, 1755 (1965).

28. Weinberg, S.: Phys. Rev. 118, 838 (1960).

29. Fink, J. P.: J. Math. Phys. 9, 1389 (1968).

K. Symanzik

Deutsches Elektronen-Synchroton DESY

D-2000 Hamburg 52, Notkestieg 1 